\title{
7
}

\section{SIMULATION OF TRAFFIC SHAPING ALGORITHMS IN ATM NETWORKS*}

\author{
M.Ajmone Marsan ${ }^{a}$, T.V.Do ${ }^{b}$, L.Jereb $^{b}$, R.Lo Cigno ${ }^{a}$, R.Pasquali $^{a}$, A.Tonietti $^{c}$ \\ ${ }^{a}$ Dipartimento di Elettronica, Politecnico di Torino, \\ Corso Duca degli Abruzzi 24, 10129 Torino - Italy \\ ${ }^{b}$ Department of Telecommunications, Technical University of Budapest, \\ Sztoczek 2, 1111 Budapest - Hungary \\ ${ }^{c}$ CSELT, Via G.Reiss Romoli 274, 10148 Torino - Italy
}

Simulation is used to investigate and compare the impact of two different traffic shaping policies on the quality of connectionless services in two ATM network topologies with various levels of traffic load. The first topology is asymmetric and bicentric, and is a possible candidate for an Italian ATM network. The second topology is symmetric and monocentric, and is a possible candidate for a Hungarian ATM network. The performance parameters collected during the execution of simulation runs comprise cell and message loss probabilities, cell and message delay jitter distributions, and the number of cells brought to destination that must be discarded because some other cells of the same message were lost.

Keyword Codes: C.2.1; I.6.3; I.6.5

Keywords: Computer-Communication Networks, Architecture and Design; Simulation and Modelling, Applications; Model Development

\section{Introduction}

The quality of service (QOS) requirements in Broadband Integrated Services Digital Networks (B-ISDNs) are very tight, both for connection-oriented (CO) and connectionless (CL) services. QOS requirements are mainly expressed in terms of cell loss probability and cell delay jitter. The former is more important for the majority of CL services, but the latter is crucial for many $\mathrm{CO}$ services.

Many performance analyses of B-ISDN have shown that acceptable QOS cannot be achieved without call admission control (CAC) mechanisms for $\mathrm{CO}$ services, and some form of control of the user behavior. It is thus necessary to implement usage parameter control (UPC) functions, that can either be preventive or repressive. Repressive UPC is performed within the network, and mainly consists in discarding cells that do not comply with the traffic contract between the user and the network. Preventive UPC is performed at the source user, and consists in functions that make sure that the traffic that crosses

\footnotetext{
${ }^{*}$ This work was performed in the framework of a research contract between Politecnico di Torino and CSELT (Centro Studi e Laboratori Telecomunicazioni), and with the support of the European Community through the PHARE-ACCORD contract n. H-9112-0353.
} 
the user-to-network interface (UNI) complies with such a contract. Preventive UPC is often called traffic shaping, while repressive UPC is often called traffic policing.

In this paper we present simulation results that show the impact of two different traffic shaping mechanisms on the QOS of CL services. Two network topologies are considered, one being essentially bicentric, and the other being essentially monocentric. The former topology is a possible candidate for an Italian ATM network, and the latter for a Hungarian ATM network.

Simulation results were obtained with a software tool named CLASS (CL ATM Services Simulator) [1], whose first version was developed at Politecnico di Torino, and is now being jointly upgraded by Politecnico di Torino and the Technical University of Budapest.

Two similar shaping policies were investigated; an adaptation of the Generic Cell Rate Algorithm (GCRA) specified in the ITU-T Recommendation I.371 [2] for traffic policing, and a Token Bucket Algorithm (TBA) [3-5].

The paper is organized as follows. In the next section we briefly outline the main features of CLASS, the simulator used in the study. Section 3 describes the structure of the considered shaping device, as well as the adopted shaping algorithms. Section 4 summarizes the main characteristics of the two networks used for the derivation of the numerical results that are presented in Section 5. A wide variety of results is enclosed and commented in the paper for the comparison of alternative solutions for traffic shaping, with different parameters and traffic loads. The numerical results allow the illustration of the impact of the features of the two networks on the gains obtained with the traffic shaping function, and the impact of shaping on the performance provided to the end user. Finally, Section 6 offers some conclusive remarks.

\section{CLASS}

CLASS [1] is a slotted synchronous simulator, entirely written in standard C language to achieve good portability.

CLASS aims at the estimation of the performances of connectionless services in ATM networks, i.e., services that are based on the exchange of messages. Messages are segmented into cells by the ATM Adaptation Layer (AAL) before transmission, and they are reconstructed before the information is delivered to the destination. Thus, the estimation of the performances of connectionless services requires the computation of performance parameters both at the cell and at the message levels.

The performance parameters computed with CLASS can be divided into three categories, as follows.

Cell and message loss probabilities - These parameters are measured considering both the whole network, and individual buffers and connections (virtual channels VCs). Loss probabilities can be further subdivided in three different contributions: the first is due to overflows in the user transmission buffers (the whole message, i.e., all of its cells, are discarded in this case); the second is due to overflows in the buffers associated with links between network nodes; the third is due to overflows in the buffers associated with the links that connect the destination node to the final destination (a user). A message is considered to be lost when at least one of its cells is lost. 
Cell and message delay jitters - Cell and message delays comprise a constant part, due to propagation delays along links, and processing delays within nodes, and of a variable part, due to the waiting time in the user transmission buffer, and to queuing in the buffers associated with links between nodes. Only the variable part is considered and measured. For each delay jitter, the histogram, the average value, and the variance are computed. Also these performance parameters are computed considering both the whole network, and individual links and VCs.

Number of useless cells brought to destination - Since the loss of only one cell causes the loss of the entire message to which the cell belongs, all the other cells of the same message that successfully reach their destination result in a useless effort by the network. It is important to count these useless cells, since their number can be greatly affected by different traffic control policies. The number of useless cells is measured considering both the whole network, and individual links and VCs. We call useless load the fraction of useless cells that are brought to destination.

The wide variety of possible application scenarios in B-ISDN translates in the need for traffic generation models of different nature, so as to adequately describe different characteristic traffic patterns. Many different types of traffic sources are available in CLASS, in order to generate a representative synthetic workload for the functional model of the ATM network operations. The user models available in CLASS are described in [1]. In the work that we report in this paper, a very simple traffic source model was used in which traffic generators produce user messages that are subsequently segmented by the AAL sublayer into cells that must be transmitted on the network. Users generate traffic whose average level and destination are controlled by a traffic matrix that specifies each individual traffic relation. The destination is randomly selected on a message basis. Messages are generated according to a Poisson arrival process. The length of the messages is distributed according to a truncated geometric with mean equal 20 cells and truncation value equal 200 cells.

\section{Shaping the user traffic}

Traffic shaping is a preventive traffic control function. It is performed close to the traffic source, in order to decrease the burstiness of the offered traffic by smoothing the flow of cells, so as to ensure that the traffic crossing the UNI meets the characteristics specified during the connection establishment phase through the CAC functions. Traffic shaping may be performed directly at the connectionless traffic source or at the LAN/WAN interworking unit.

The traffic shaping function is thus interposed between the traffic source and the node of the ATM network, and it can be combined with some fair queuing service algorithms such as the Virtual Spacing Scheduling proposed by Roberts [10] in order to guarantee a minimal service rate for best effort services.

The shaping algorithm considered in this paper differs from other algorithms described in the literature, since it is specifically designed to suit the connectionless traffic environment where cells are generated in batches. Instead of discarding cells as in the case of policing or in the case of the cell spacer described in [7], our shaping algorithm delays cells 


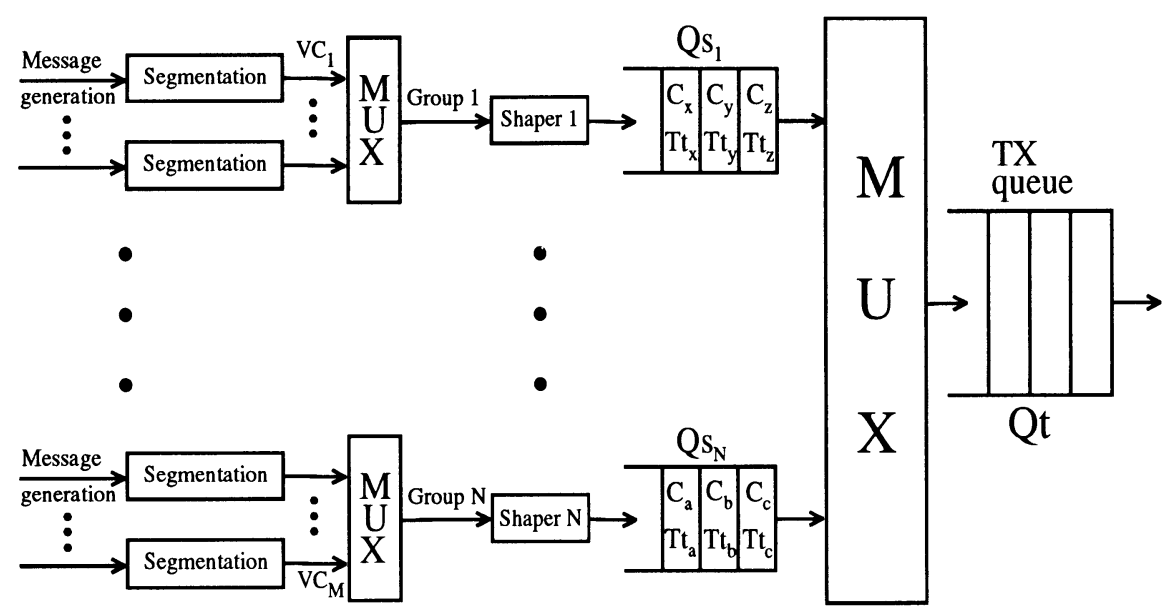

Figure 1. Architecture for traffic shaping

until they comply with the traffic contract. In the cell spacer described in [7-9], Boyer et al. proposed the insertion of a spacing function after the policing function at the UNI and NNI (network node interface) in order to decrease the CDV (cell delay variation) of connection-oriented traffic which may become intolerable when a circuit emulation has to be performed. Therefore, in their device only the conforming cells are spaced.

The architecture of the traffic shaping device that we have considered is depicted in Fig. 1. Message generations correspond to the invocation of AAL service primitives from higher layer protocols. Messages are then segmented into cells, and batches of cells corresponding to whole messages are forwarded to the shapers, that implement a specified shaping algorithm on the traffic referring to a given group of VCs. When more than one $\mathrm{VC}$ belongs to a group, multiplexing the VCs into groups is necessary before shaping. Batches of cells (deriving from one message) are multiplexed at this stage. A further multiplexing of the shaped cell streams is necessary before crossing the UNI when more than one group is defined at a user. Single cells are multiplexed in this second multiplexer.

Several different architectures may be devised for the implementation of the traffic shaping functions, and their performances may significantly differ. A quantification of the relative merits of different shaping approaches is left for further study.

\subsection{Multiplexing VCs into groups}

As we noted before, the shaping function is applied to groups of VCs that originate from a common user; thus, messages belonging to a given group must be multiplexed before shaping.

Many different groupings of VCs can be defined. At one extreme, each VC can be individually shaped; at the other extreme, all the VCs originating from one user are collectively shaped. As intermediate solutions, different groupings of VCs can be considered. 
In principle, VCs can be grouped in any arbitrary way, but a specific performance objective should suggest to group the VCs on the basis of some common characteristics, such as the destination node, the path through part of the network, or other.

\subsection{The shaping algorithms}

Two similar shaping policies were investigated. The first policy is an adaptation of the Generic Cell Rate Algorithm (GCRA) specified in the ITU-T Recommendation I.371 [2] for traffic policing. While there the GCRA is recommended for cell marking or discarding, the adaptation that we consider delays cells until they are considered compliant by the algorithm. The second policy is a shaping version of a simple leaky bucket, where again cells are delayed rather than discarded; similar to what is done in the literature, we refer to this policy with the name Token Bucket Algorithm (TBA) [3-5].

Both the GCRA and the TBA depend on two key parameters.

1. The bandwidth allocated to the group of VCs on which a shaper operates; this parameter defines the token generation rate for the TBA, and the nominal cell interarrival time, denoted by $T$, in the GCRA.

2. The allowed cell burst length; this parameter defines the token buffer size for the TBA and the cell delay variation tolerance for the GCRA. The token buffer size for the TBA is denoted by $T B S$; the cell delay variation tolerance for the GCRA is denoted by $\tau$.

For both algorithms, the goal is to compute the allowed transmission time for an incoming cell, given its theoretical arrival time, and its actual arrival time.

- The actual arrival time of cell $C_{i}, T a_{i}$, is the generation time of the message to which $C_{i}$ belongs.

- The theoretical arrival time of cell $C_{i}, T A T_{i}$, is determined as a function of the nominal interarrival time, and of the actual arrival times of previous cells.

- The allowed transmission time of cell $C_{i}, T t_{i}$, is the time at which $C_{i}$ is eligible for transmission.

Cells arriving at a shaper are tagged with a time stamp equal to the message generation time, that is used for shaping and cell delay jitter computation. The allowed transmission time $T t_{i}$ for each cell $C_{i}$ of the VCs in the $n$-th group is computed by the $n$-th shaper which places the cells into its output queue $Q s_{n}$, together with the computed value of $T t_{i}$.

Note that, due to the presence of the output multiplexer, the allowed transmission time of $C_{i}$ in general does not coincide with its actual transmission time, except for the case in which the shaper operates only on one group of VCs.

\subsubsection{The GCRA shaper}

The GCRA shaper is defined by the following algorithm to be executed at every time slot for each shaper operating on a group of VCs. TIME identifies the current slot.

if $T a_{i}=T I M E$

- if $T a_{i}>T A T_{i}$

then $T t_{i}=T a_{i}, \quad T A T_{i+1}=T a_{i}+T$

- if $T A T_{i}-\tau \leq T a_{i}<T A T_{i}$ then $T t_{i}=T a_{i}, \quad T A T_{i+1}=T A T_{i}+T$

- if $T a_{i}<T A T_{i}-\tau$ then $T t_{i}=T A T_{i}-\tau, \quad T A T_{i+1}=T A T_{i}+T$ 


\subsubsection{The TBA shaper}

The TBA shaper is defined by the following algorithm to be executed at every time slot for each shaper operating on a group of VCs. The admission of every cell into the ATM network requires the removal of one token from the bucket. TIME identifies the current slot; $N T$ is the number of tokens in the token bucket; $T_{n t}$ is the future time slot when the first token (not reserved to a queued cell), will arrive into the token buffer.

The algorithm is made of two steps that must be executed in the given order.

- Step 1:

$$
\begin{array}{clll}
\text { if } T_{n t}=T I M E & & \\
\text { - then } & N T=N T+1 & & \\
\text { - if } & N T<T B S & \text { then } & T_{n t}=T_{n t}+T \\
\text { - if } & N T=T B S & \text { then } & T_{n t}=\infty
\end{array}
$$

- Step 2:

$$
\begin{aligned}
& \text { if } T a_{i}=T I M E \\
& \text { - if } \quad N T=T B S \quad \text { then } T t_{i}=T a_{i}, \quad N T=N T-1, \quad T_{n t}=T a_{i}+T \\
& \text { - if } 0<N T<T B S \text { then } T t_{i}=T a_{i}, \quad N T=N T-1 \\
& \text { - if } N T=0 \quad \text { then } T t_{i}=T_{n t}, \quad T_{n t}=T_{n t}+T
\end{aligned}
$$

Any TBA shaper can always be mapped onto a GCRA shaper by letting

$\tau=T(T B S-1)$

Instead, a GCRA shaper in which $\tau$ is not a multiple of $T$ cannot be mapped onto a TBA shaper.

\subsection{Multiplexing after the shaper}

At each user, the number of shapers equals the number of groups of VCs. If more than one shaper is present, their outputs must be multiplexed in order to enqueue the cells for transmission on the link leading to the node. Only when all the VCs originating at one user are collectively shaped, no multiplexer is necessary, since only one shaper exists.

The multiplexing step before the transmission buffer is fairly critical, because it may result in quite a negative effect on the burstiness of the cell streams, thus nullifying much of the shaper work. A great care is thus necessary in the design of the multiplexing algorithms, whose impact on the traffic profile on the link may be substantial.

In the simulation models, we have adopted a multiplexing algorithm similar to the one described in [6]. With reference to Fig. 1, define the following notation:

- $Q t$ : is the transmission queue associated with the link leading to the ATM node

- $Q s_{n}$ : is the queue at the output of the $n$-th shaper

- $C_{i}:$ is a generic cell

- $T t_{i}$ : is the allowed transmission time for cell $C_{i}$

- $T I M E$ : as always, indicates the current slot 
The multiplexer operation at any slot follows the algorithm described by the following pseudo code:

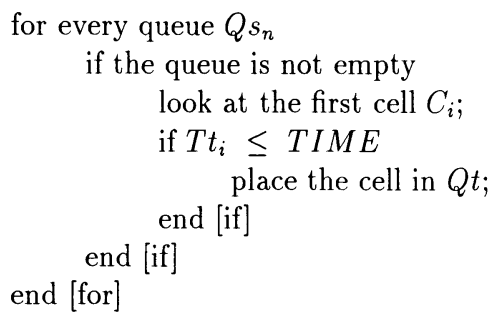

The shaper queues are visited in a deterministic order, with the aim of reducing the randomness of delays between cells of the VCs of the same group. Cells from queue $Q t$ are transmitted in a FIFO order.

\section{The two networks}

The performance study was carried out on two different network topologies, which are possible candidates for an Italian ATM network, and for a Hungarian ATM network, respectively. For this reason, the two topologies will be labeled $I$ and $H$.

The two topologies share some common features. They comprise a similar number of nodes (10 for $I$ and 9 for $H$ ), users (19 for $I$ and 21 for $H$ ), and bidirectional links (17 for $I$ and 14 for $H$ ). In our study, the two networks are assumed to have identical buffer sizes inside each node (100 cell positions for every link), and to use identical shaping policies. The traffic in the two network models is generated by the same types of users (Poisson message generation with truncated geometric message length distribution, with average 20 cells, and truncation value 200 cells).

From other points of view the two topologies are different. The $I$ topology is bicentric and asymmetric, whereas the $H$ topology is monocentric and symmetric. The sum of the capacities of links in the $I$ network is $8.7 \mathrm{Gbit} / \mathrm{s}$, resulting from 8 links at $600 \mathrm{Mbit} / \mathrm{s}$, and 26 links at $150 \mathrm{Mbit} / \mathrm{s}$. The sum of the capacities of links in the $H$ network is 4.2 Gbit/s, resulting from 28 links at $150 \mathrm{Mbit} / \mathrm{s}$. In both networks, $300 \mathrm{Mbit} / \mathrm{s}$ channels are obtained with two $150 \mathrm{Mbit} / \mathrm{s}$ links in parallel.

The overall traffic load is higher for the $I$ network, and so are the (average and peak) loads at the UNI, while inside the network the average link load is higher in the $H$ network.

Fig. 2 shows the topology of the $I$ network, and Fig. 3 shows the topology of the $H$ network.

The traffic patterns that define the workload in the two networks are given in Table 1 for the $I$ network, and in Table 2 for the $H$ network. The matrices define the traffic load on a node-by-node basis; the node traffic is then equally distributed among the users connected to the node. The last row of each traffic matrix gives the total traffic generated by the users connected to the node.

The traffic is not uniformly distributed in the $I$ network, but it is uniformly distributed in the $H$ network with the outstanding role of the center node (node 1). 


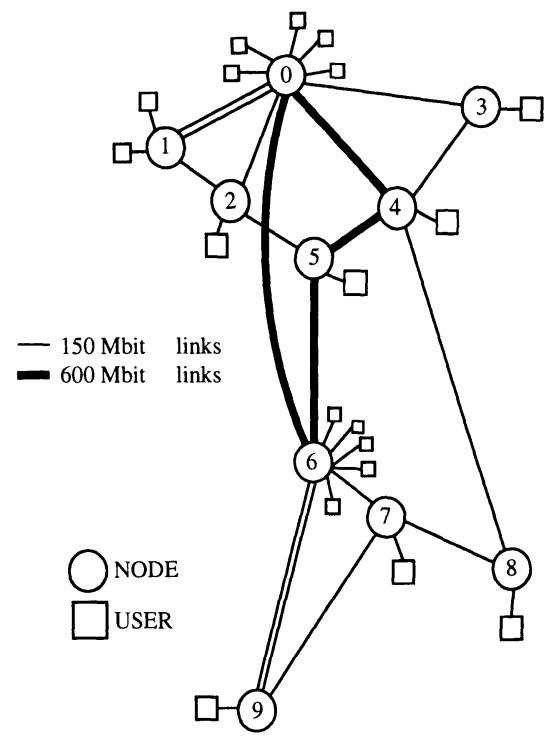

Figure 2. Topology of the $I$ network

\begin{tabular}{|c||r|r|r|r|r|r|r|r|r|r|}
\hline Node & 0 & 1 & 2 & 3 & 4 & 5 & 6 & 7 & 8 & 9 \\
\hline \hline 0 & 0 & 52 & 20 & 2 & 18 & 3 & 206 & 17 & 2 & 17 \\
\hline 1 & 52 & 0 & 3 & 0 & 3 & 5 & 32 & 3 & 0 & 3 \\
\hline 2 & 20 & 3 & 0 & 0 & 2 & 2 & 12 & 1 & 0 & 1 \\
\hline 3 & 2 & 0 & 0 & 0 & 0 & 0 & 1 & 0 & 0 & 0 \\
\hline 4 & 18 & 3 & 2 & 0 & 0 & 2 & 11 & 1 & 0 & 1 \\
\hline 5 & 35 & 5 & 2 & 0 & 2 & 0 & 22 & 2 & 0 & 2 \\
\hline 6 & 206 & 32 & 12 & 1 & 11 & 22 & 0 & 10 & 1 & 10 \\
\hline 7 & 17 & 3 & 1 & 0 & 1 & 2 & 10 & 0 & 0 & 1 \\
\hline 8 & 2 & 0 & 0 & 0 & 0 & 0 & 1 & 0 & 0 & 0 \\
\hline 9 & 17 & 3 & 1 & 0 & 1 & 2 & 10 & 1 & 0 & 0 \\
\hline \hline Total & 369 & 101 & 41 & 3 & 38 & 70 & 305 & 35 & 3 & 35 \\
\hline
\end{tabular}

Table 1

Traffic matrix for the $I$ network; the traffic is generated by the node in the column and goes to the node in the row; the relations are expressed in thousandths of the global generated traffic 


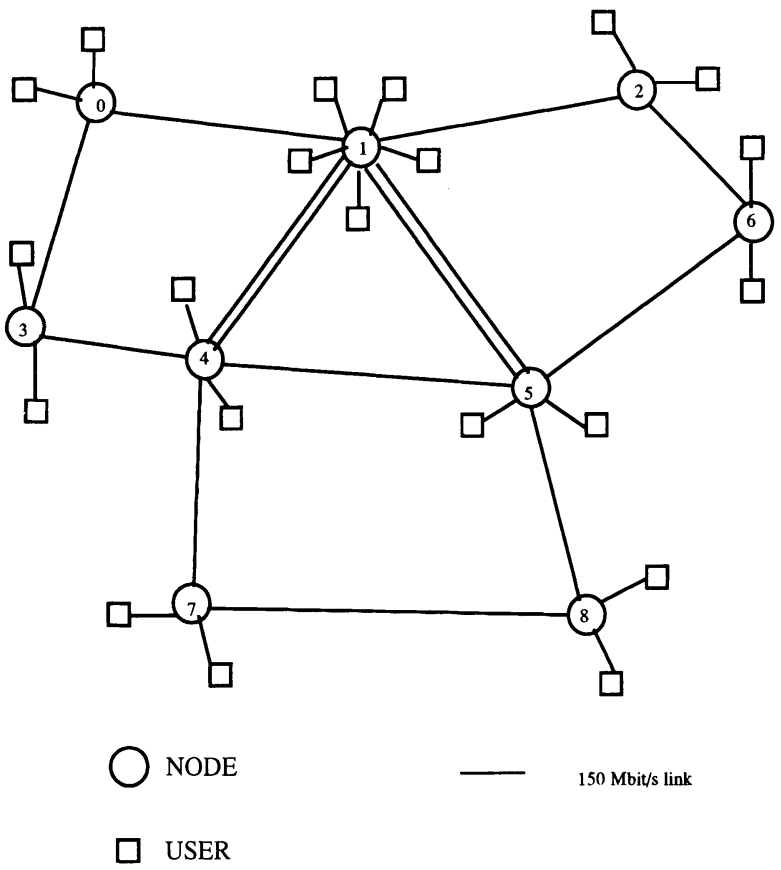

Figure 3. Topology of the $H$ network

\begin{tabular}{|c||r|r|r|r|r|r|r|r|r|}
\hline Node & 0 & 1 & 2 & 3 & 4 & 5 & 6 & 7 & 8 \\
\hline \hline 0 & 0 & 45 & 5 & 5 & 5 & 5 & 5 & 5 & 5 \\
\hline 1 & 45 & 0 & 45 & 45 & 45 & 45 & 45 & 45 & 45 \\
\hline 2 & 5 & 45 & 0 & 5 & 5 & 5 & 5 & 5 & 5 \\
\hline 3 & 5 & 45 & 5 & 0 & 5 & 5 & 5 & 5 & 5 \\
\hline 4 & 5 & 45 & 5 & 5 & 0 & 5 & 5 & 5 & 5 \\
\hline 5 & 5 & 45 & 5 & 5 & 5 & 0 & 5 & 5 & 5 \\
\hline 6 & 5 & 45 & 5 & 5 & 5 & 5 & 0 & 5 & 5 \\
\hline 7 & 5 & 45 & 5 & 5 & 5 & 5 & 5 & 0 & 5 \\
\hline 8 & 5 & 45 & 5 & 5 & 5 & 5 & 5 & 5 & 0 \\
\hline \hline Total & 80 & 360 & 80 & 80 & 80 & 80 & 80 & 80 & 80 \\
\hline
\end{tabular}

Table 2

Traffic matrix for the $H$ network; the traffic is generated by the node in the column and goes to the node in the row; the relations are expressed in thousandths of the global generated traffic 


\begin{tabular}{|c||c|c|}
\hline Grouping & $I$ & $H$ \\
\hline \hline VCG & 300 & 384 \\
\hline DNG & 143 & 168 \\
\hline FBG & 71 & 74 \\
\hline SUG & 19 & 21 \\
\hline
\end{tabular}

Table 3

Total number of groups in the $I$ and $H$ networks for the different groupings

\section{Numerical results}

In this section we present some simulation results obtained for the two networks described in Section 4 with the shaping algorithms illustrated in Section 3.

Four groupings of VCs will be considered, and labeled as follows.

VCG - (VC grouping) the traffic on each VC is individually shaped.

DNG - (destination node grouping) the traffic is shaped grouping all the VCs originating in one user, and whose destination users are connected to the same node.

FBG - (first bifurcation grouping) the traffic is shaped grouping all the VCs originating in one user, and still sharing the same link after crossing the first node.

SUG - (source user grouping) the traffic on all the VCs that originate in one user is collectively shaped.

The implementation of SUG is trivial, since no distinction among cells is necessary. The implementation of VCG requires the inspection of the VCI field in each cell, and appears to be simple.

The DNG and FBG were mainly considered for comparison; their implementation may be non trivial, due to the need to identify the group to which an arbitrary cell belongs. Possibly, VPIs could be used for this purpose, but introducing such a restriction should be justified by significant performance gains.

Table 3 shows the total number of groups in the $I$ and $H$ networks for the four different considered groupings.

The bandwidth allocated to each group of VCs $\left(B A_{n}\right.$ for the $n$-th group) defines the nominal cell interarrival time for the two shaping algorithms ( $T_{n}$ for the $n$-th group).

If $B_{n i}$ is the average bandwidth of the $i$-th $\mathrm{VC}$ of the $n$-th group, $B A_{n}=\beta \sum_{i} B_{n i}$, where $\beta$ is the "bandwidth allocation factor", that in our experiments is taken to be constant for all the groups in the network. From $B A_{n}, T_{n}$ is obtained as

$T_{n}=\max \left(1,\left\lfloor\frac{C}{B A_{n}}\right\rfloor\right)$

where $C$ is the data rate of the link connecting the source user to the first node of the ATM network. $C$ always equals $150 \mathrm{Mbit} / \mathrm{s}$ in our simulation experiments. 


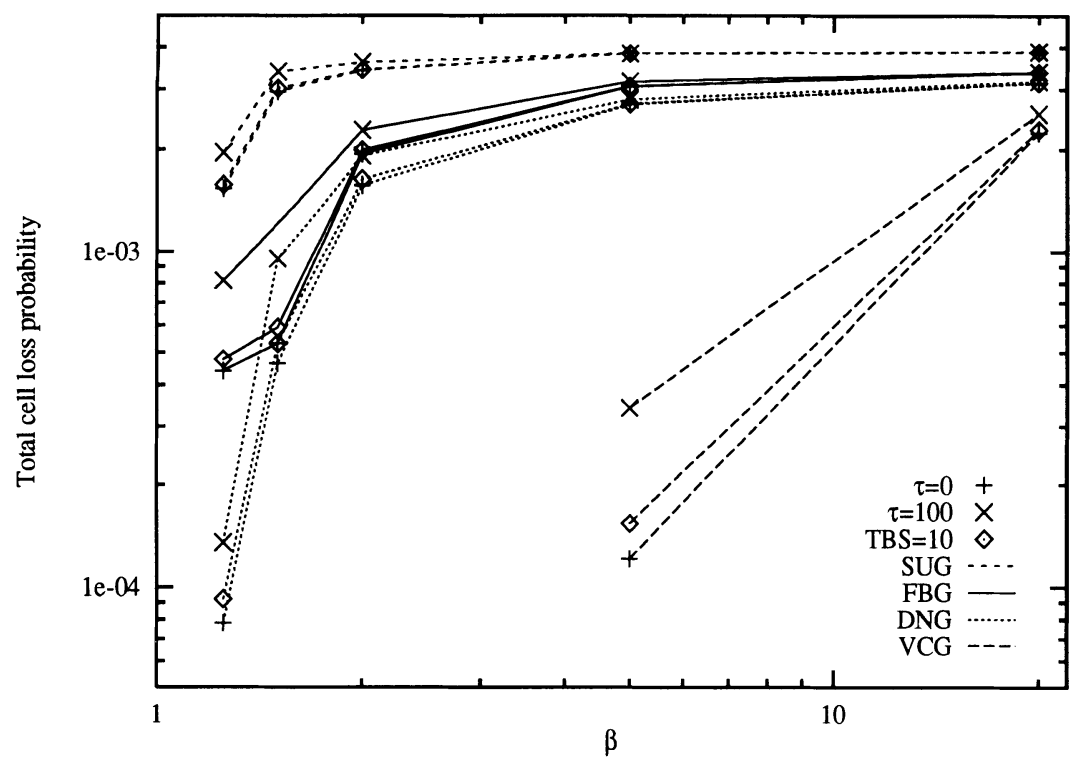

Figure 4. Total cell loss probability versus the bandwidth allocation factor $\beta$ in the $I$ network with a total load equal to $800 \mathrm{Mbit} / \mathrm{s}$

In order to obtain comparable results for the delay jitters in the two networks, all times will be normalized to the slot duration on $150 \mathrm{Mbit} / \mathrm{s}$ links.

For the GCRA, two values for the cell delay variation tolerance $\tau$ are initially considered: $\tau=0$, and $\tau=100$. Later on, also the value $\tau=20$ will be used. Note that with $\tau=0$ all cells of the $n$-th group are spaced by at least $T_{n}$ slots. For the TBA, the token buffer size is always taken to be 10 . However, the results for the GCRA with $\tau=0$ coincide with those that would be obtained for the TBA with $T B S=1$.

For most of the results presented in this paper, the total traffic in the $I$ network equals $800 \mathrm{Mbit} / \mathrm{s}$, while in the $H$ network equals $600 \mathrm{Mbit} / \mathrm{s}$.

The size of the user buffers was chosen large enough to guarantee that no message loss occurs; this means that no cells are lost at the source user. Instead, as we already mentioned, the size of all buffers inside the two networks is taken to be 100 cells, and when these buffers overflow, cell losses occur.

\subsection{The impact of shaping}

Figs. 4 and 5 show, for the $I$ and $H$ networks, respectively, curves of the total cell loss probability (probability of cell loss at any buffer within the ATM network nodes, including the buffer associated to the final link, connecting the destination node to the destination user) versus the bandwidth allocation factor $\beta$, for the four considered groupings. The shaping algorithms are the GCRA with $\tau=0$ and 100, and the TBA with TBS=10. Simulations were run for values of $\beta$ equal to $1.25,1.5,2,5,10$, and 20 . No cell loss events were observed for those values of $\beta$ where cell loss probabilities are not reported. 


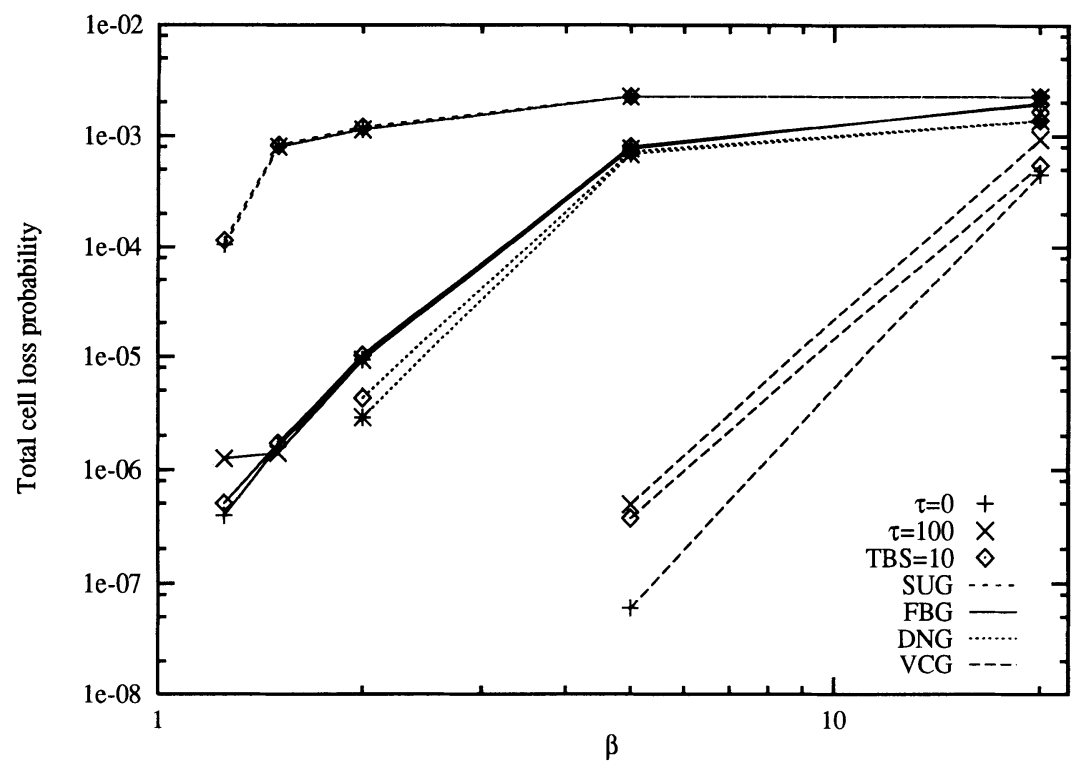

Figure 5. Total cell loss probability versus the bandwidth allocation factor $\beta$ in the $H$ network with a total load equal to $600 \mathrm{Mbit} / \mathrm{s}$

Cell loss probabilities were always estimated through simulation, with no extrapolation, and confidence intervals were always provided by CLASS for all performance parameters. In most cases the confidence intervals are quite narrow, since a significant number of cell loss events were observed before stopping the simulation experiments. Furthermore, confidence intervals can be obtained even in those cases when no cell loss is observed. It is also worth emphasizing that most simulation runs require less than one hour of CPU time on an IBM RISC/6000 570 workstation running AIX.

The main goal of the shaping function is the improvement of the ATM network performance in terms of cell loss probability. This is achieved with all the considered groupings of VCs and shaping algorithms, but with great differences in efficiency. It is quite evident from the figures that VCG provides the largest advantages, and that small values of $\beta$ are a necessity. Furthermore, smaller values of $\tau$ (or $T B S$ ) are preferable, but the influence of this parameter on the cell loss probability is not as large. In particular, the SUG approach seems to provide marginal improvements, which are visible only for small values of $\beta$. The two groupings DNG and FBG seem to behave in a way that is not satisfactory enough to overcome the possible implementation difficulties.

A special comment is necessary in order to explain the strange behaviour of the curves of FBG for low values of $\beta$ in the $I$ network. Indeed, only in the case of FBG, a reduction of the value of $\beta$ from 1.5 to 1.25 induces a marginal reduction of the cell loss probability. This happens because for this grouping (and only for it with the given input data) the reduction of $\beta$ has quite a marginal impact on the values of $T_{n}$ for groups with high traffic. This is due to the quantization effect resulting from the floor operator in (2). In practice, 


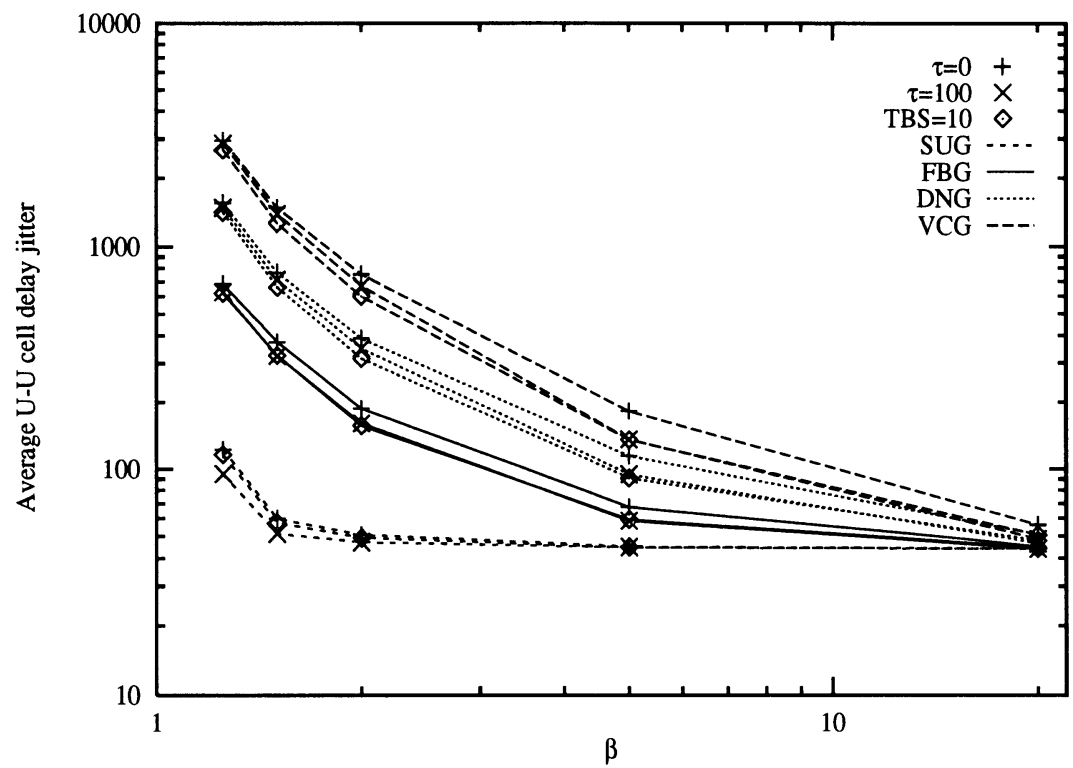

Figure 6. Average user-to-user cell delay jitter versus the bandwidth allocation factor $\beta$ in the $I$ network with a total load equal to $800 \mathrm{Mbit} / \mathrm{s}$

what happens is that some groups with low traffic ( $T_{n}$ from about 10 to 100$)$ are affected by the change in the values of $\beta$, but groups with high traffic $\left(T_{n}\right.$ equal to 1 or 2$)$ do not experience any change.

The previous results lead to the conclusion that the grouping of VCs is quite important, and that the larger the number of groups, the better; hence, VCG seems to be the correct approach, and that rather small values of $\beta$ should be used.

If we turn our attention to Figs. 6 and 7, where curves of the average user-to-user cell delay jitter (variation of the delay from the message generation instant until the cell delivery at the destination user) are plotted versus $\beta$, we see that the price that has to be paid for the reduction of the cell loss probability is a large increase in the average user-to-user cell delay jitter. When VCG is used with small values of $\beta$, the increase can be two orders of magnitude! Of course, other groupings (SUG in particular), and large values of $\beta$, can produce much smaller increments of the average user-to-user cell delay jitter.

Another interesting observation is that, since the spacing parameter $T$ is not identical for all groups of VCs, the different impact of GCRA and TBA results in intersections of the curves. However, these differences are not significant, thus from now on we will only present results for GCRA, using $\tau=20$.

The increase in the average user-to-user cell delay jitter is due to a longer sojourn in the source user buffer, waiting for admission into the ATM network. Indeed, if we jointly look at the curves of the average user-to-user cell delay jitter and average cell delay jitter inside the network (Figs. 8 and 9), we see that the latter decreases similarly to the cell 


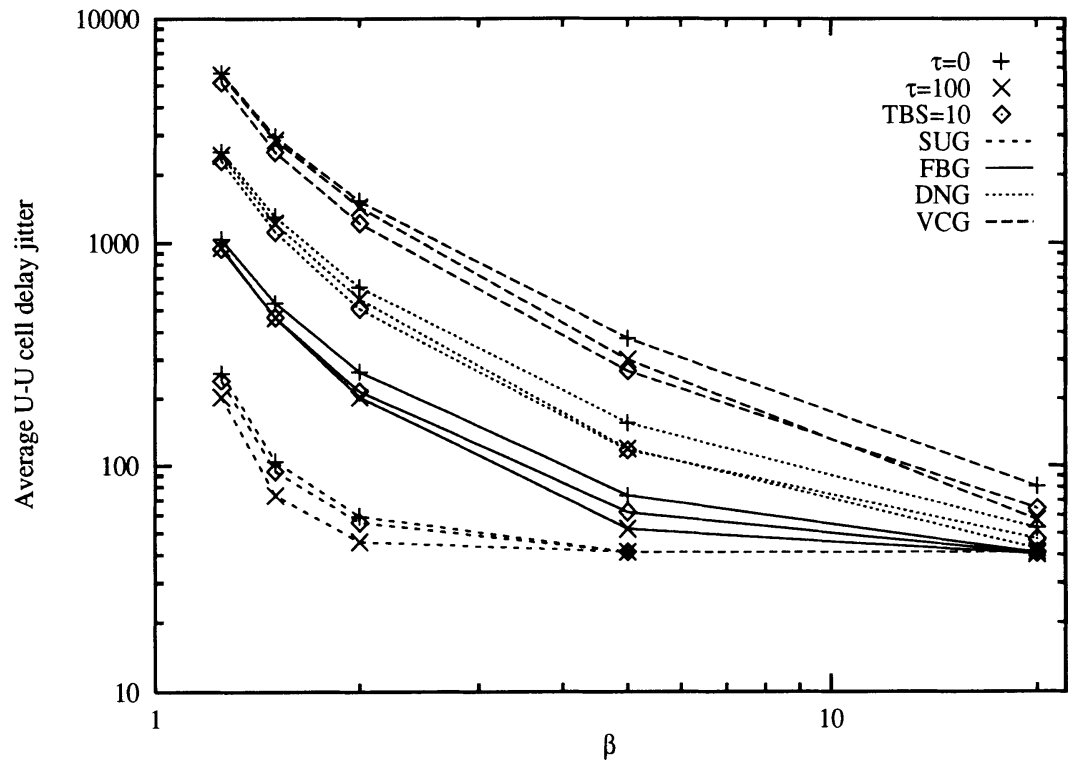

Figure 7. Average user-to-user cell delay jitter versus the bandwidth allocation factor $\beta$ in the $H$ network with a total load equal to $600 \mathrm{Mbit} / \mathrm{s}$

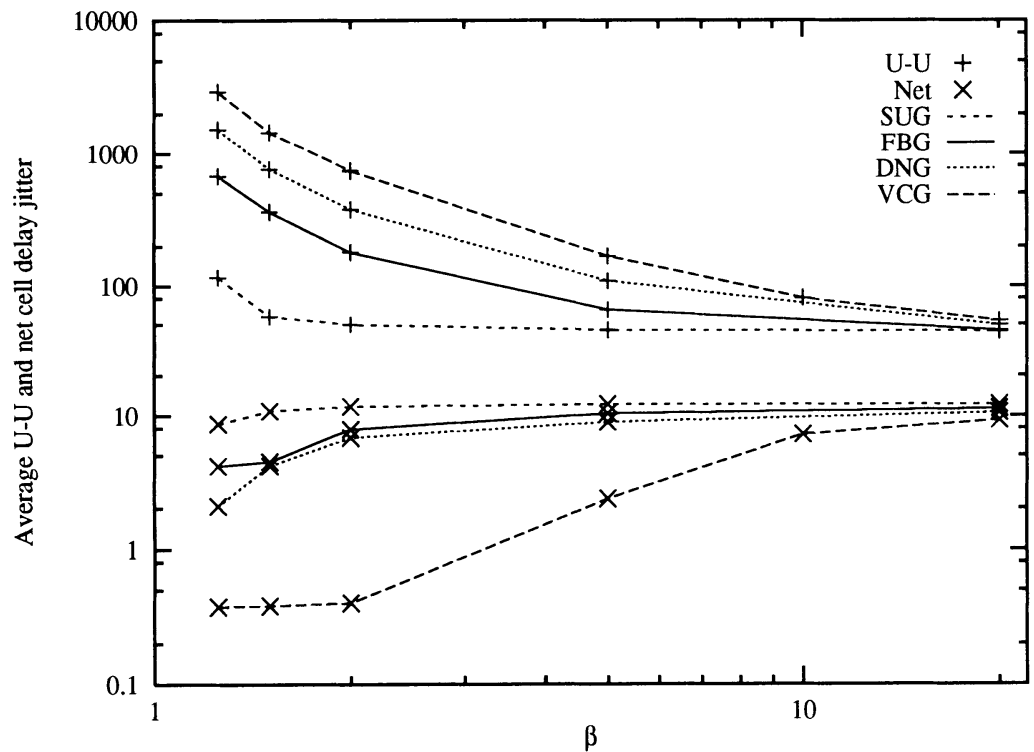

Figure 8. Average cell delay jitter inside the $I$ network together with average user-to-user cell delay jitter versus the bandwidth allocation factor $\beta$, for a total load equal to 800 $\mathrm{Mbit} / \mathrm{s}$ and with $\tau=20$ 


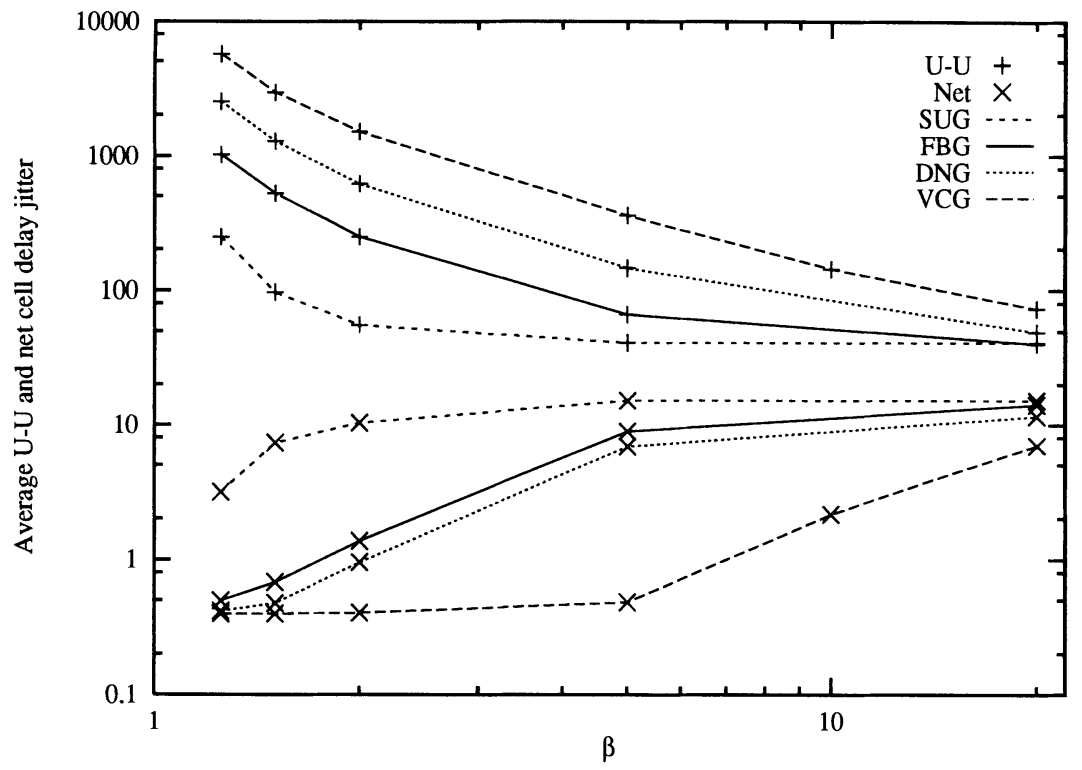

Figure 9. Average cell delay jitter inside the $H$ network together with average user-to-user cell delay jitter the bandwidth allocation factor $\beta$, for a total load equal to $600 \mathrm{Mbit} / \mathrm{s}$ and with $\tau=20$

loss probability. The difference between the user-to-user cell delay jitter and the cell delay jitter inside the network is exactly the time spent by a cell in the source user buffer; its average is measured by the vertical distance between the two curves with the same line type in Figs. 8 and 9 , and it can be seen to grow with the number of shapers, and for decreasing values of $\beta$.

The sojourn time in the source user buffer is due to queuing in front of the transmitter; when no traffic shaping is performed, this queuing time can be low, but it obviously increases when a shaper reduces the rate at which cells are allowed to enter the ATM network.

The results observed in Figs. 8 and 9 are rather obvious, since a tighter shaping of the input traffic produces a better behaviour inside the network, which results in improvements in terms of both cell loss probability, and average cell delay jitter inside the network, but in a longer average user-to-user delay jitter.

In Figs. 10 and 11, two further interesting characteristics of the impact of shaping are plotted. Fig. 10 shows curves of the coefficient of variation of the user-to-user cell delay jitter (standard deviation over average), versus the average of the same parameter, for the two networks, and the four groupings of VCs. In both networks the curves can be observed to initially increase, and then to flatten out. In Fig. 11, similar curves are plotted, referring to the cell delay jitter inside the two networks. The behavior in this case is different, since a maximum can be observed in all curves (note that, in order to show this peak in the $H$ network with VCG grouping, an additional simulation experiment was 


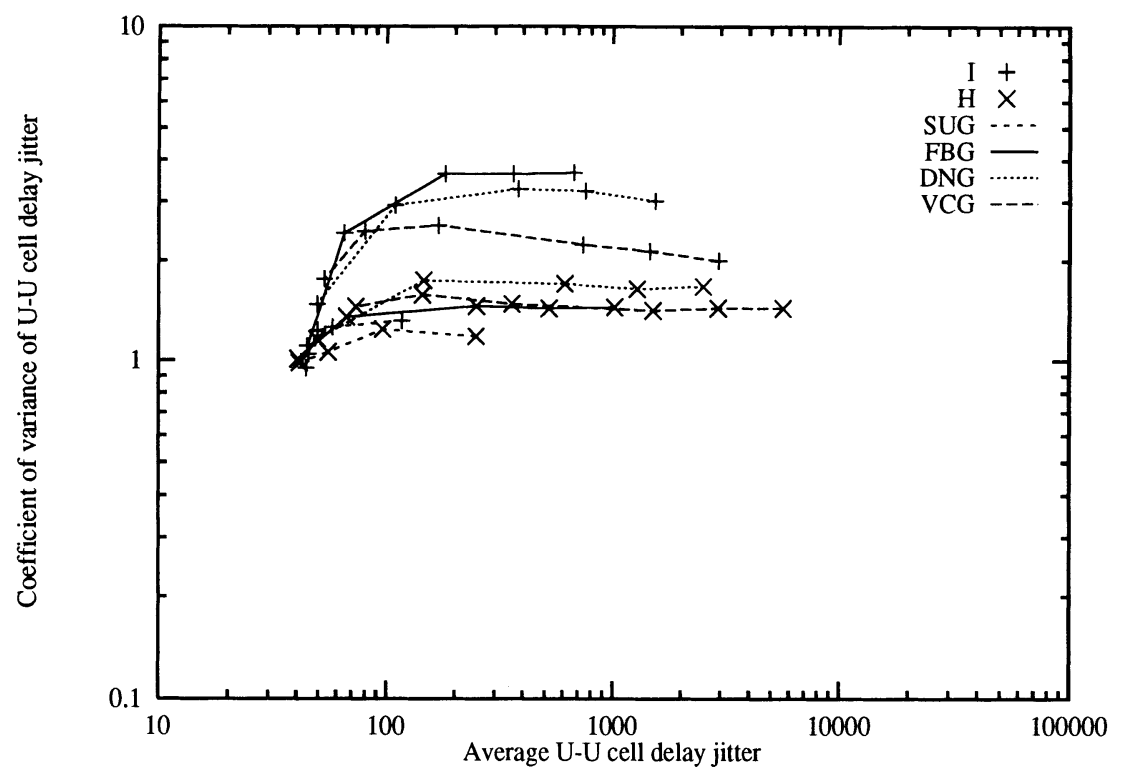

Figure 10. Coefficient of variation of the user-to-user cell delay jitter versus average user-to-user cell delay jitter for the two networks with $\tau=20$

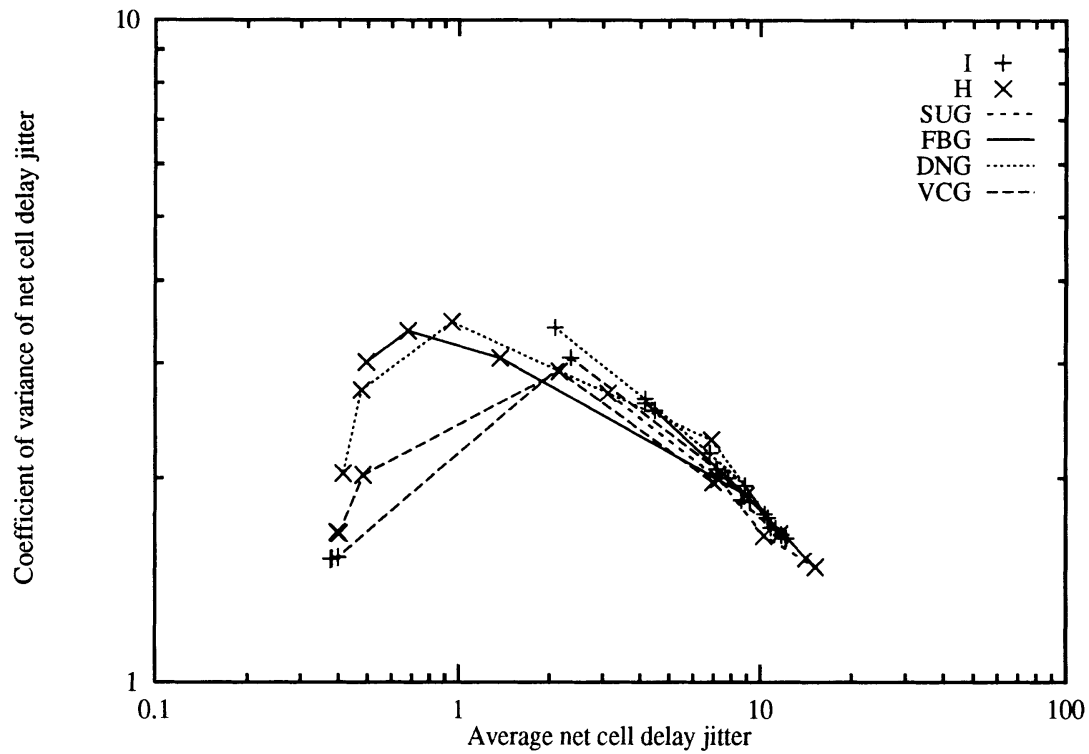

Figure 11. Coefficient of variation of the cell delay jitter inside the network versus average cell delay jitter inside the two networks with $\tau=20$ 


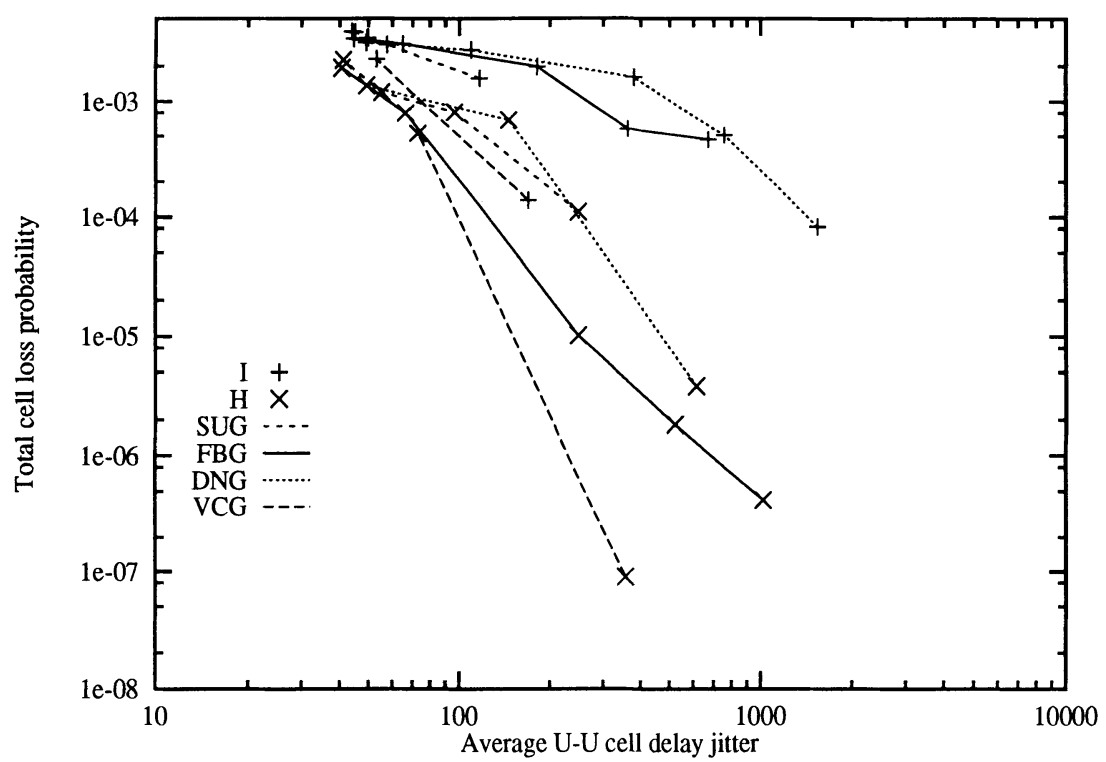

Figure 12. Cell loss probability versus the average user-to-user cell delay jitter for the two networks and the four groupings

performed with $\beta=10$.)

These results are not surprising: when shaping tightly controls the admission of cells into the network, the user-to-user cell delay jitter is high, but the cell delay jitter inside the network is low. The flow of cells is very smooth inside the network, so that the coefficient of variation of the cell delay jitter inside the network is low. However, source user buffers are heavily loaded because cells must wait for a significant amount of time before being allowed to enter the ATM network, so that the coefficient of variation of the user-to-user cell delay jitter is high. When the cell admission control enforced by shaping becomes looser, the user-to-user cell delay jitter decreases, but the cell delay jitter inside the network grows. The occupancy of buffers inside the network is quite variable, and the coefficient of variation of the cell delay jitter inside the network grows. The time spent in the source user buffers before admission into the network is reduced, however its variability remains large, and the coefficient of variation of the user-to-user cell delay jitter does not change significantly. Finally, when hardly any shaping is performed, the buffers inside the network are often full, some cells with the longest delay are lost, so that the coefficient of variation of the cell delay jitter inside the network decreases again. The time spent in the source user buffers becomes short, and the coefficient of variation of the user-to-user cell delay jitter decreases significantly.

In Fig. 12 we show the curves of the cell loss probability versus the average user-to-user cell delay jitter for the two networks and the four groupings. These curves are quite interesting for the comparison of the different groupings, since we have on the horizontal axis the price to be paid in terms of the user-to-user cell delay jitter, and on the vertical 


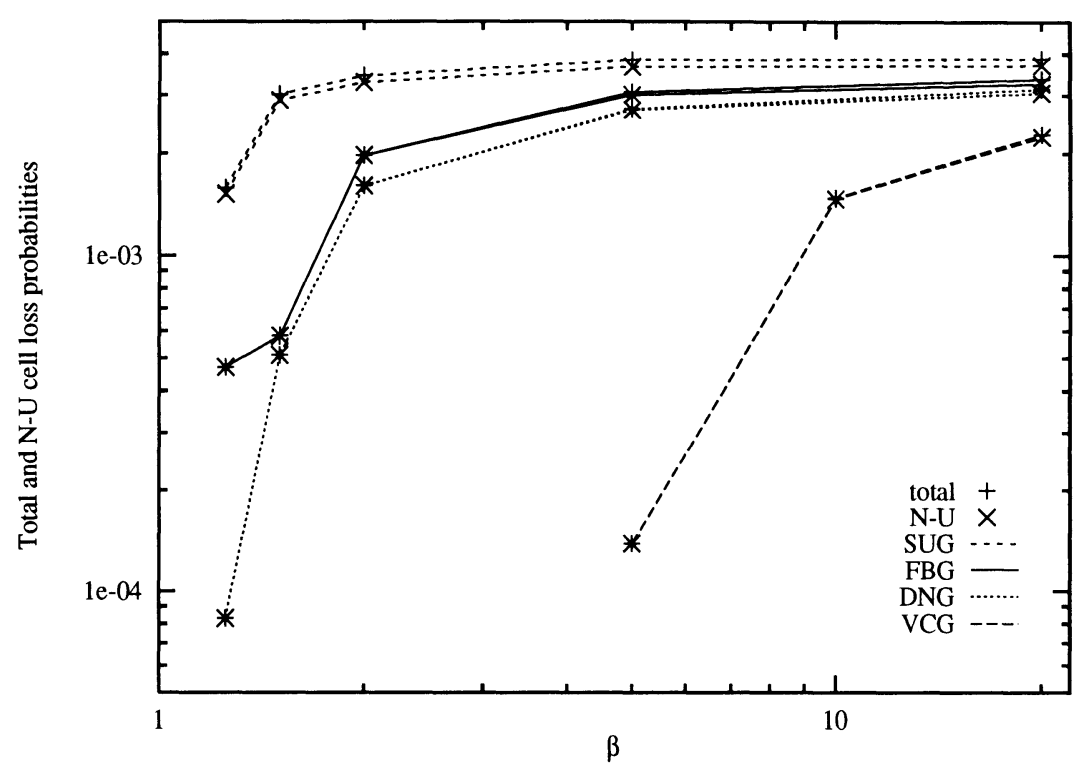

Figure 13. Total and N-U cell loss probabilities versus the bandwidth allocation factor $\beta$, in the $I$ network for a total load equal to $800 \mathrm{Mbit} / \mathrm{s}$ and with $\tau=20$

axis the gain in terms of the cell loss probability. Of course, the better the shaping policy, the more its points remain low and to the left. From these results the impact of the different groupings is more clear, and we can conclude once more that VCG is the most effective grouping, and that the two intermediate groupings do not provide performance gains that justify their implementation complexity.

\subsection{The bottlenecks in the $I$ network}

We now turn our attention to the comparison of the $I$ and $H$ networks. Several observations are possible from the plots that were presented so far. The behaviors of the different shaping policies in the two networks appear to be fairly similar, however, some significant differences exist.

On the one hand, the increase in the average user-to-user cell delay jitter can be seen to be similar for the two networks. Instead, although the range of the values is almost identical some differences exist for the reduction of the average cell delay jitter inside the networks, but these do not clearly favor one of the two networks. On the other hand, the $I$ network shows higher values and smaller reductions of cell loss probabilities, moreover higher values in the coefficient of variation can also be observed. Some of the differences in the coefficient of variation can be explained by the fact that the $H$ network is symmetric, with users injecting similar amounts of traffic into the network, while in the $I$ network the input traffic varies significantly for the different users.

The large number of losses in the $I$ network is mainly due to the bottleneck effect occurring when cell streams traveling on a $600 \mathrm{Mbit} / \mathrm{s}$ link must be forwarded on a 150 


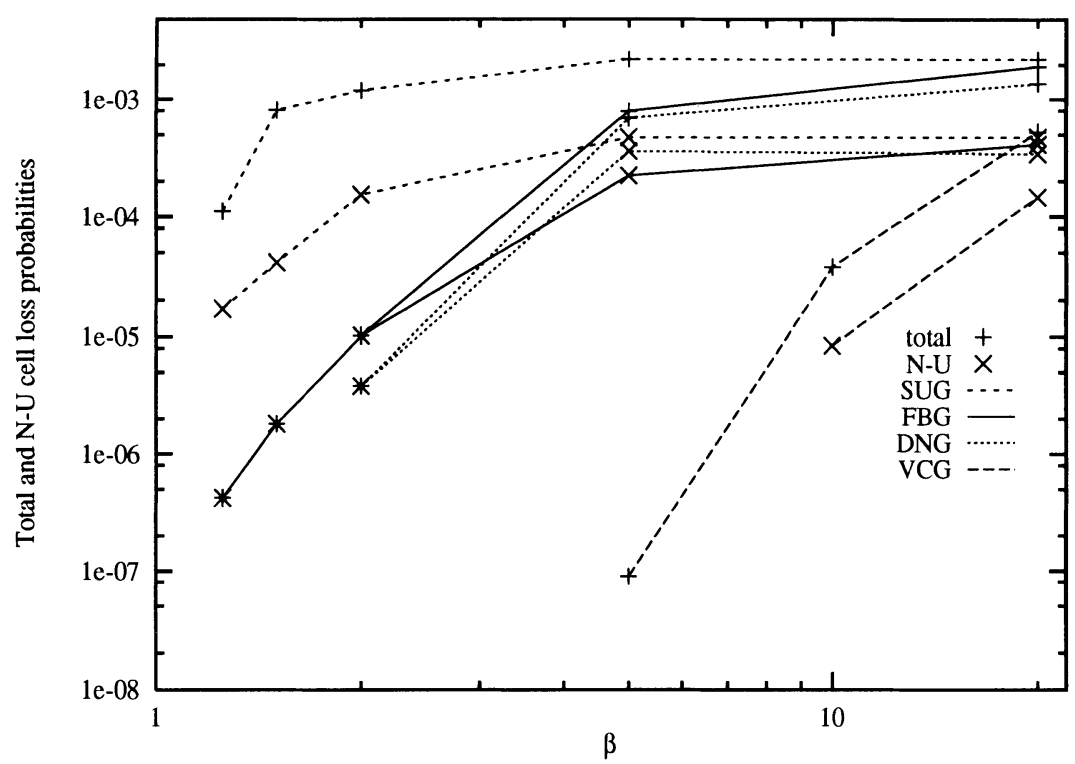

Figure 14. Total and N-U cell loss probabilities versus the bandwidth allocation factor $\beta$, in the $H$ network for a total load equal to $600 \mathrm{Mbit} / \mathrm{s}$ and with $\tau=20$

Mbit/s link. The effect of this bottleneck is illustrated by the results in Figs. 13 and 14, where the total cell loss probability curves are shown together with the curves of the loss probability in the buffers associated with the link connecting the destination node with the destination user ( $\mathrm{N}-\mathrm{U}$ loss), for the $I$ and $H$ networks, respectively. In Fig. 13, the curves show that in the $I$ network a vast majority of the cell losses (as well as the delay jitter inside the network) take place within the buffers connecting destination nodes to destination users, and this is true in particular at those destination nodes that can be reached with a $600 \mathrm{Mbit} / \mathrm{s}$ link. This effect is stressed even more by the fact that in the $I$ network the $600 \mathrm{Mbit} / \mathrm{s}$ links carry a large fraction of the traffic.

Instead, in the $H$ network, where all links have the same data rate and such a bottleneck effect is not present, when the cell loss probability is significant, cell losses are more evenly distributed in all the network buffers. The cell loss probability can be reduced by more than three orders of magnitude in the observable range, and even larger reductions are certainly achieved for those cases in which no losses were observed. Fig. 14 clearly shows this behavior, since for large values of the bandwidth allocation factor $\beta$, the $\mathrm{N}$-U loss only amounts to about $10 \%$ of the total loss. For small values of $\beta$, no cell loss occurs also inside the $H$ network.

Another interesting observation is that the results in the $H$ network clearly demonstrate that the cell shaping performed at the source has a much greater impact on the cell loss probability inside the network rather than on the cell loss probability at the buffer between the destination node and the destination user. This is due to the fact that the multiplexing stages inside the network can drastically alter the characteristics of the traffic. In other 


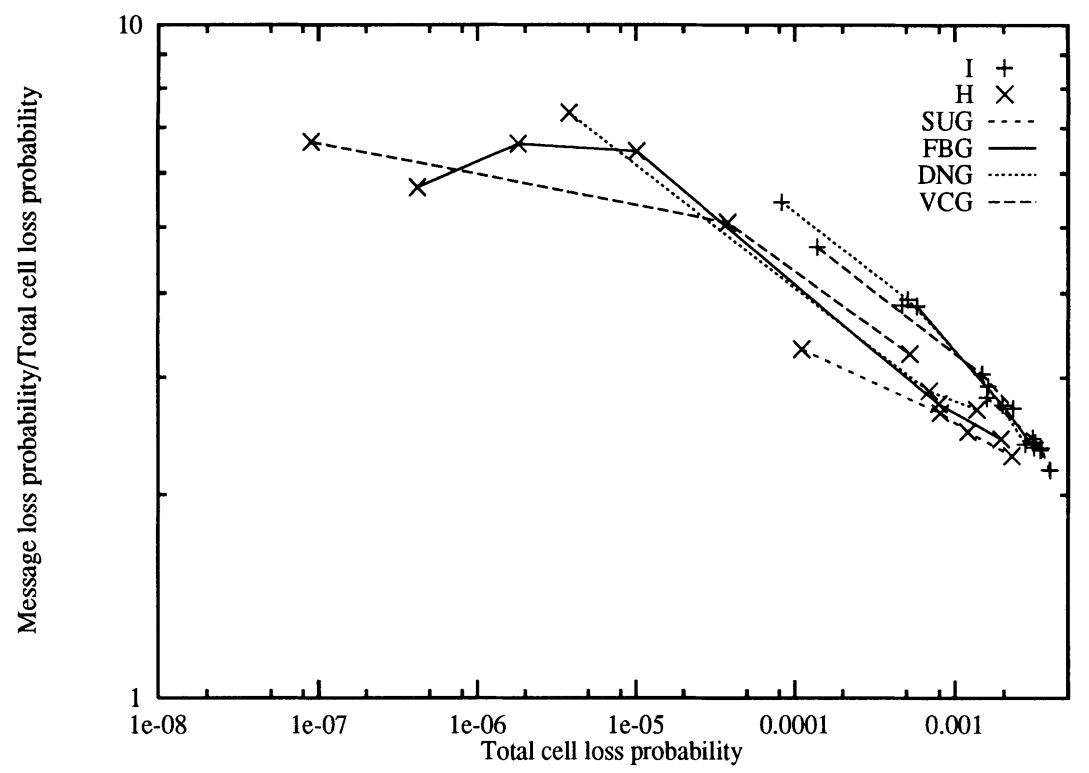

Figure 15. Message loss probability over cell loss probability versus the cell loss probability for the two networks and the four groupings

words, if we focus onto a specific buffer in the network, and onto the loss probability in this buffer, the closer is the buffer to the shaper, the more effective is the shaping function on the loss probability in this buffer.

\subsection{Message-level performance}

Results concerning message loss probabilities and message delay jitters are crucial in the evaluation of the performances of connectionless services. At first sight, the results available for the message loss probability and the message delay jitter show a behaviour very similar to the same parameters referred to cells. For this reason, in order to reduce the number of figures to be presented, we only describe some interesting features of the message-level quantitative results.

In Figs. 15 and 16 we plot the curves of the ratio between the message loss probability and the total cell loss probability as well as the curves of the ratio between the number of useless cells brought to destination and the number of cells lost, versus the cell loss probability for the two networks and the four groupings, respectively. As expected, the two figures behave similarly: in both cases a higher burstiness of the cell loss events reduces the value of the ratio.

It is important to observe that the ratios decrease for increasing cell loss probability. This result is due to the fact that when cell losses inside the network are very rare, messages are discarded because of the loss of a small number of cells and at the destination a greater number of cells must be discarded for each cell lost at any buffer inside nodes of the ATM network; hence we can conclude that the correlation among cell loss events is 


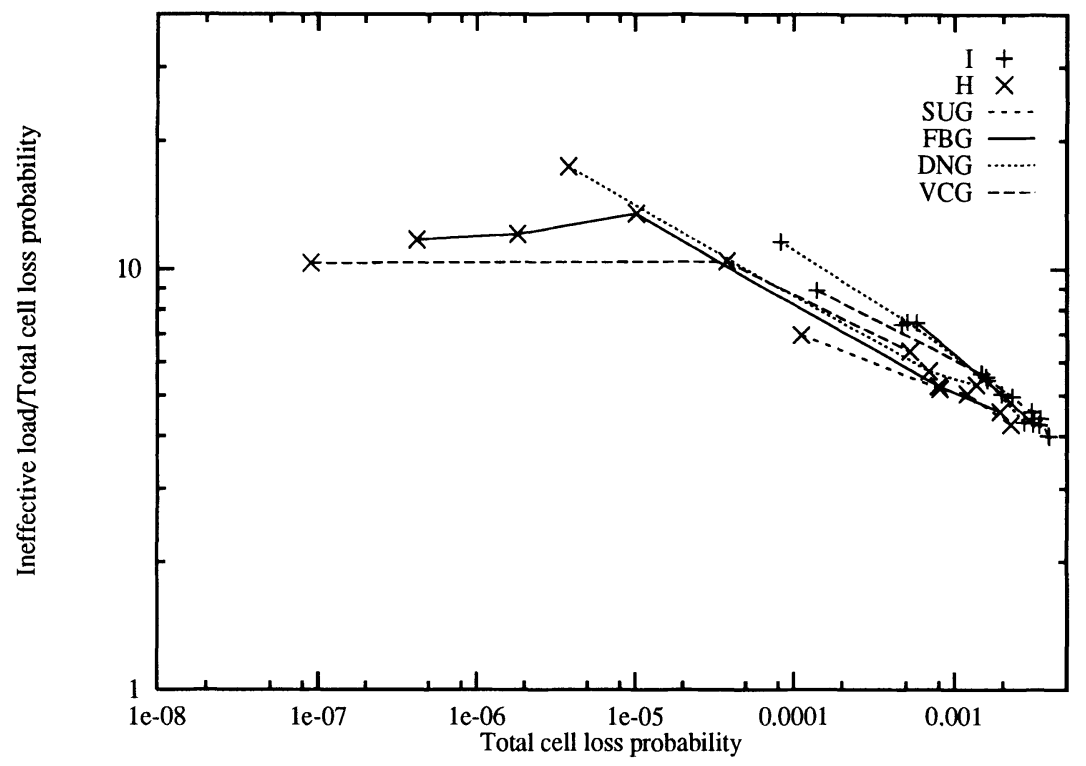

Figure 16. Number of useless cells brought to destination over number of cells lost versus the cell loss probability for the two networks and the four groupings

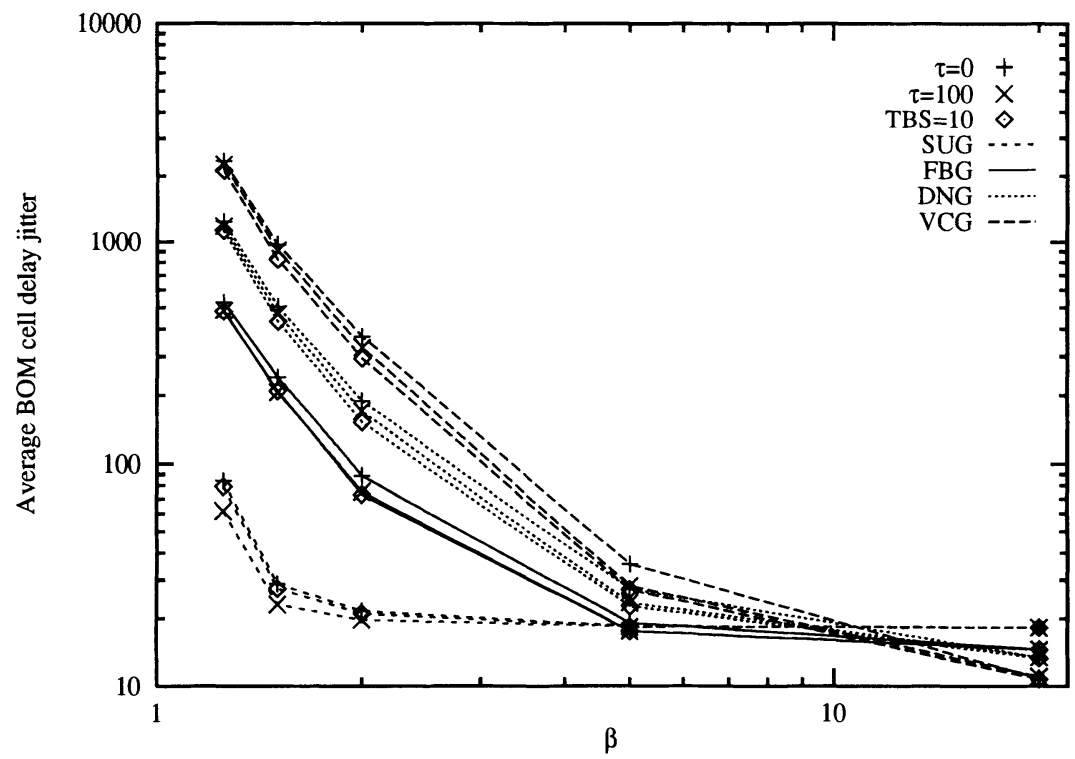

Figure 17. Average Begin Of Message cell delay jitter versus the bandwidth allocation factor $\beta$, in the $I$ network for a total load equal to $800 \mathrm{Mbit} / \mathrm{s}$ 


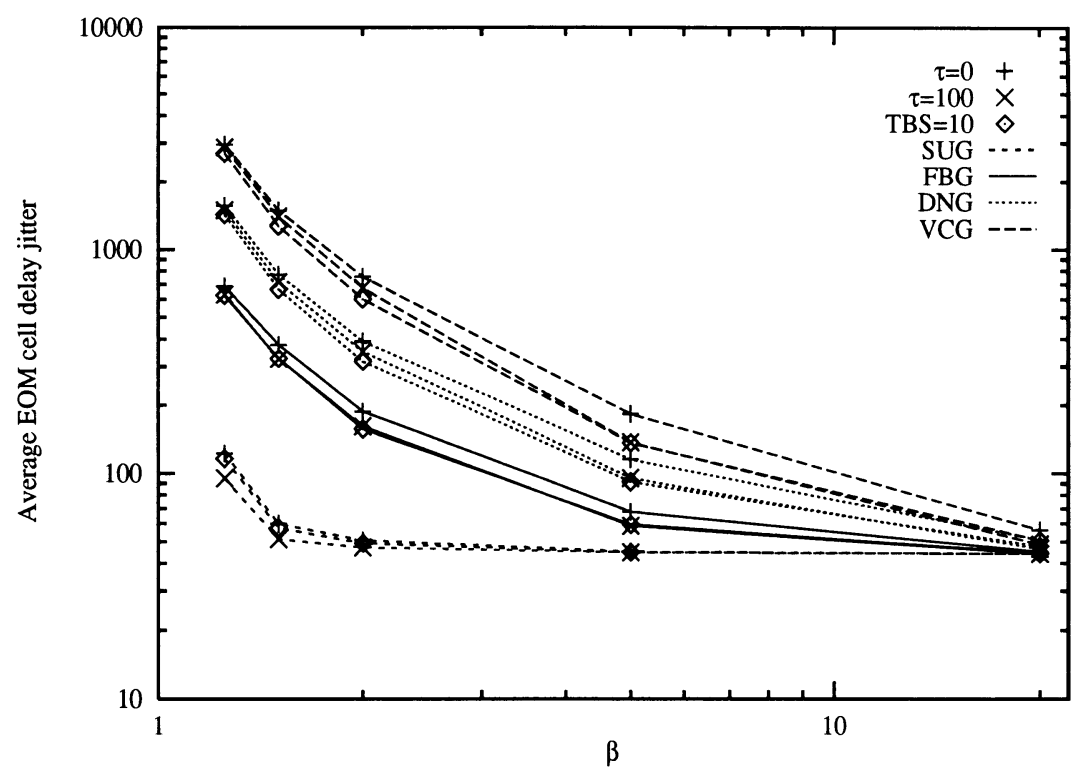

Figure 18. Average End Of Message cell delay jitter versus the bandwidth allocation factor $\beta$, in the $I$ network for a total load equal to $800 \mathrm{Mbit} / \mathrm{s}$

quite small. Instead, when the cell loss probability is high, the correlation among cell loss events grows, and a large number of cells of the same message are lost inside the network in one burst of cell loss events; thus relatively less messages are lost and less cells have to be discarded at the destination user.

In Figs. 17 and 18 we plot two new parameters, the average BOM (Begin Of Message) and EOM (End Of Message) cell delay jitters, versus the bandwidth allocation factor $\beta$, with $\tau=0,100$ and $T B S=10$. The curves show results similar to those presented for the user-to-user cell delay jitter, as expected. However, Fig. 17 indicates an interesting feature of the shaping function; when large values of the bandwidth allocation factor are used, the average BOM delay jitter decreases because the presence of a larger number of groups allows the admission into the network of the BOM cell of a new message before the cells of a message that arrived earlier and that were spaced according to the allowable bandwidth. This may have a significant impact on the reassembly function at the destination.

\subsection{The impact of the traffic load}

After the illustration of the impact of shaping on the different performance parameters, we present some numerical results obtained with variable traffic load. In Figs. 19, 20, and 21 , the total cell loss probability, the average user-to-user cell delay jitter, and the average cell delay jitter inside the network are plotted for the two networks with $\beta=2$ and $\tau=20$, respectively.

In Fig. 19 we can see that the total cell loss probability is rapidly increasing with increasing load, as expected. The growth is more pronounced in the $H$ network, rather 


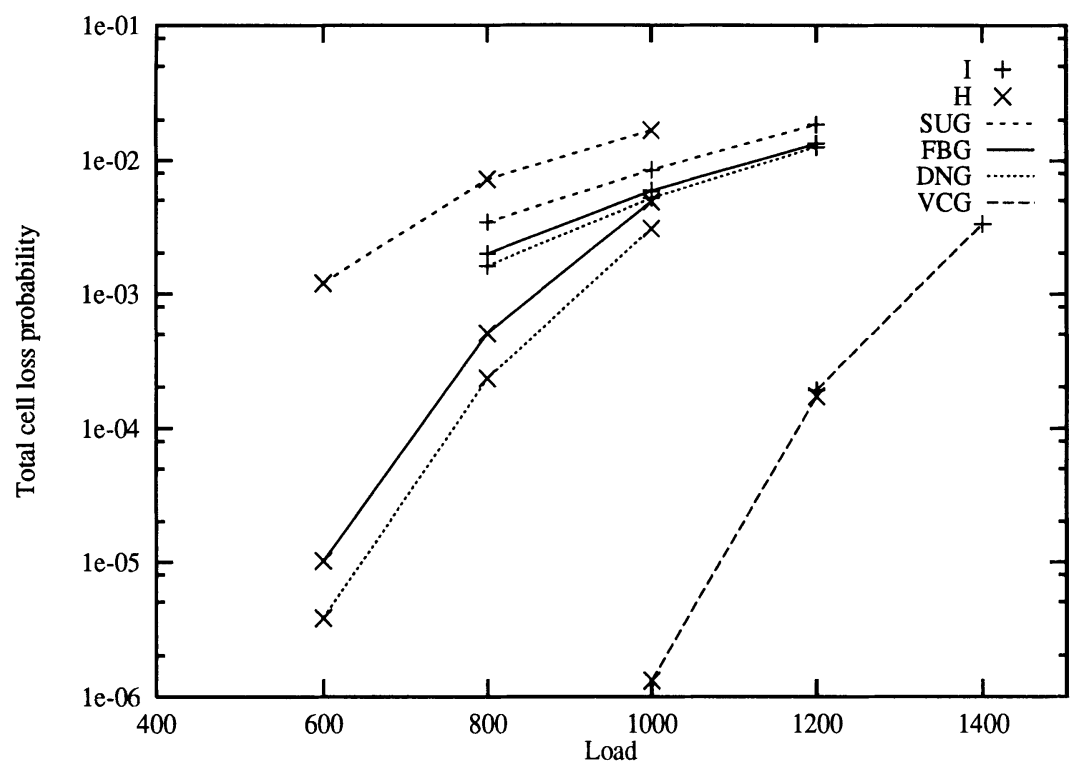

Figure 19. Total cell loss probability versus total traffic load for the two networks and the four groupings, with $\beta=2$ and $\tau=20$

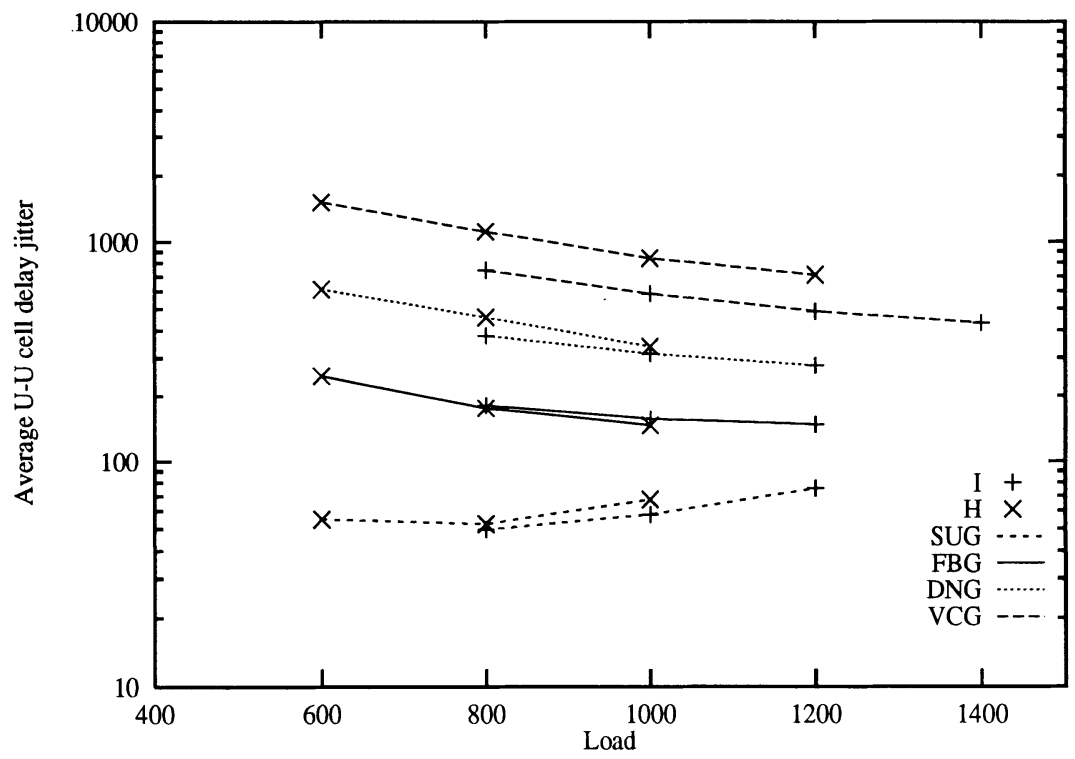

Figure 20. Average user-to-user cell delay jitter versus total traffic load for the two networks and the four groupings, with $\beta=2$ and $\tau=20$ 


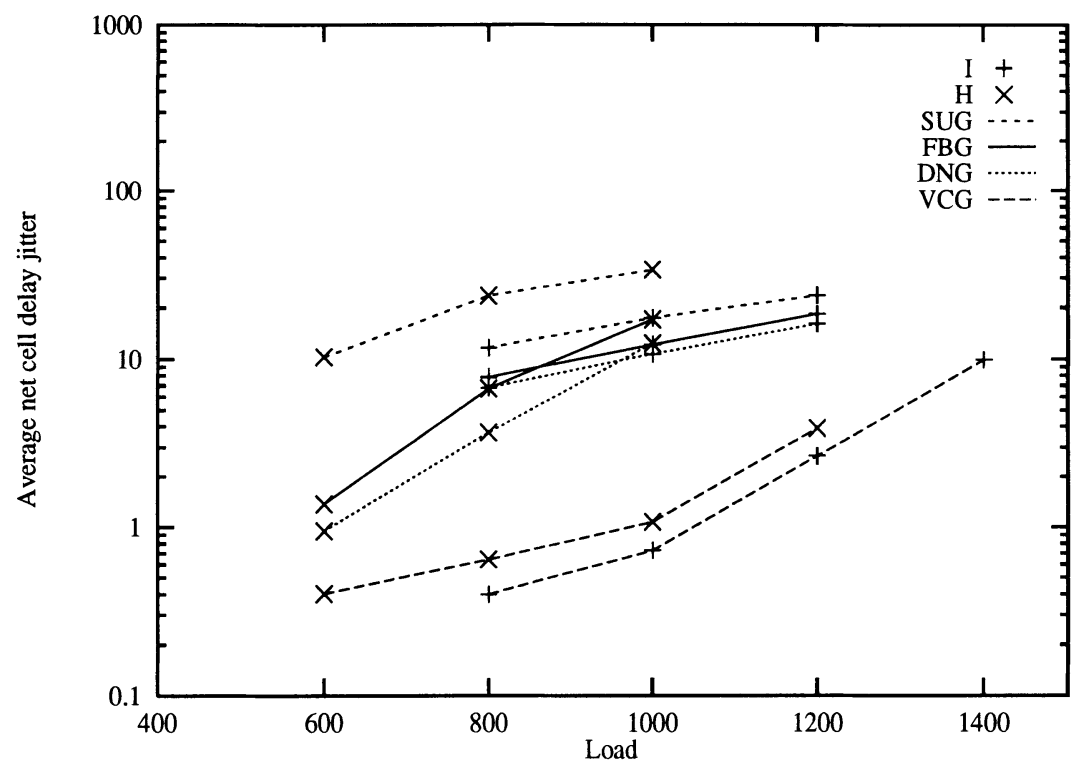

Figure 21. Average cell delay jitter inside the two networks versus the total traffic load for the four groupings, with $\beta=2$ and $\tau=20$

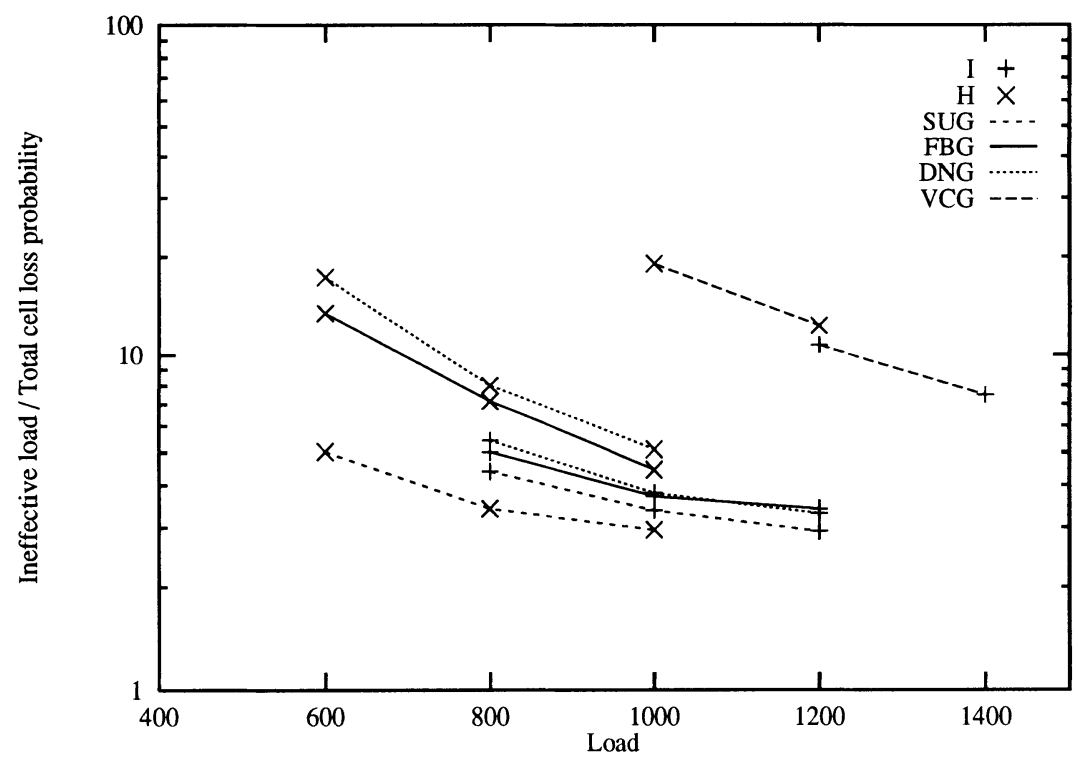

Figure 22. Useless load over user-to-user cell loss probability versus the total traffic load for the two networks and the four groupings, with $\beta=2$ and $\tau=20$ 
than in the $I$ network. This is due to the fact that the traffic in the $H$ network is more uniform than in the $I$ network. Thus, when the total traffic load grows, in the $I$ network the cell loss probability increases only for a limited number of traffic relations, whereas the whole $H$ network is brought closer to saturation. This also means that in the $I$ network cell losses still happen mainly in the buffers connecting the destination nodes to the destination users, whereas in the $H$ network for increasing load the presence of losses in the internal network buffers becomes predominant.

The behaviour of the average user-to-user cell delay jitter in Fig. 20 is surprising, since a (slight) decrement of this parameter can be observed for increasing load. The explanation of this feature derives from the fact that when the traffic increases, so does the bandwidth allocated to groups of VCs, and shaping allows cells to enter into the network more rapidly, thus reducing the cell sojourn time in the source user buffer.

The curves of the average cell delay inside the network are plotted in Fig. 21; the behaviour looks similar to that of the total cell loss probability, and seems very intuitive.

Results for the ratio of the useless load over the total cell loss probability versus the total traffic load are shown in Fig. 22. The curves behave like in Fig. 15: with increasing load (and increasing cell loss probability) the useless load decreases, because of the higher burstiness of cell loss events.

\subsection{The impact of the traffic mix}

The previous sections always presented aggregate performance measures, averaging over the whole network, and thus disregarded the effects of the traffic distribution, which can be very significant, specially when the traffic load is unevenly distributed, like in our $I$ network.

A detailed study of the impact of the traffic distribution on the network performance requires enormous amounts of CPU time, and is left for further study, but some interesting results were already obtained that can provide the reader with a flavour of the behaviours that can be observed.

In Fig. 23 we show the cell delay jitter in the shaper versus the spacing parameter $T$, for different mean message interarrival times $(M M I T)$, chosen equal to the values of the minimum, maximum and average $M M I T$ of messages in the $I$ network. The results in Fig. 23 are derived through an exact analytical model described in [11].

On the one hand, the curves in Fig. 23 show that the cell delay jitter in the user buffer can widely vary with the $\mathrm{VC}$ load, specially when the spacing parameter $T$ is large: differences can grow larger than one order of magnitude. Thus, delay jitters can become extremely high for users with low bit rate. On the other hand, the availability of the results in Fig. 23 allows the shaper parameters to be dimensioned so as to provide "fair" conditions for the different users.

Consider the case of VCG shaping only, for simplicity. Up to now we always assumed a "bandwidth-fair" approach in which all VCs are given identical $\beta$ values. It is also possible to consider a "spacing-fair" case, where all VCs are given identical $T$ values, and a "delay-fair" case, in which the shaper parameters are selected so as to make the cell delay jitter in the shaper similar for all VCs. The "delay-fair" case can be dimensioned using the results in Fig. 23.

In Table 4 we report some numerical results for the three "fair" cases. Two groups of 


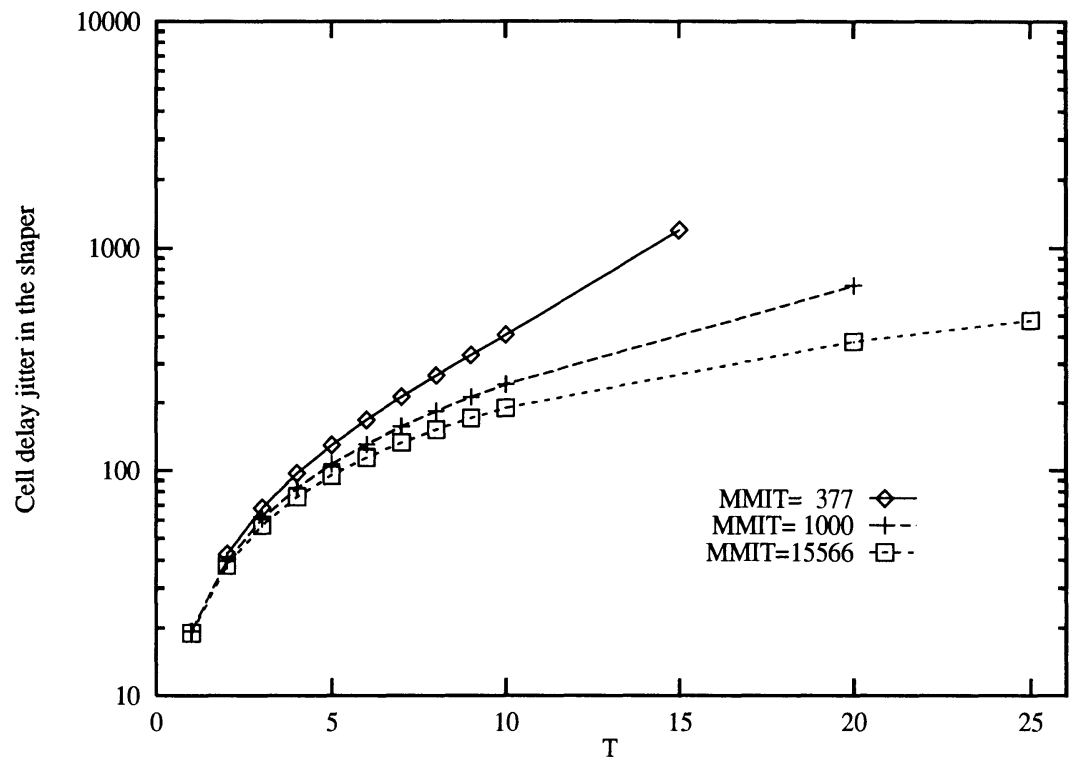

Figure 23. Cell delay jitter in the shaper versus the spacing parameter $T$, for different mean message interarrival times (MMIT) with average message length 20

\begin{tabular}{|c||c|c|c|}
\hline & "Bandwidth-fair" & "Spacing-fair" & "Delay-fair" \\
\hline \hline average $T$ & 7.7 & 7.0 & 7.0 \\
\hline minimum $T$ & 3 & 7 & 6 \\
\hline maximum $T$ & 155 & 7 & 9 \\
\hline ASCDJ & 182 & 183 & 174 \\
\hline $\begin{array}{c}\text { coefficient of variation } \\
\text { of ASCDJ }\end{array}$ & 1.57 & 0.15 & 0.054 \\
\hline minimum ASCDJ & 69 & 116 & 150 \\
\hline maximum ASCDJ & 4302 & 220 & 207 \\
\hline \hline average $T$ & 20.5 & 14.0 & 17.7 \\
\hline minimum $T$ & 9 & 14 & 13 \\
\hline maximum $T$ & 389 & 14 & 39 \\
\hline ASCDJ & 762 & 684 & 770 \\
\hline $\begin{array}{c}\text { coefficient of variation } \\
\text { of ASCDJ }\end{array}$ & 1.49 & 0.46 & 0.055 \\
\hline minimum ASCDJ & 311 & 233 & 663 \\
\hline maximum ASCDJ & 19352 & 1183 & 925 \\
\hline
\end{tabular}

Table 4

Impact of the traffic mix on the average shaper cell delay jitter (ASCDJ) in the $I$ network 
results are reported. In both cases the parameters of the shapers were selected trying to obtain similar average shaper cell delay jitters (ASCDJ) as aggregate parameters. In the "bandwidth-fair" case, the values of $\beta$ were set to $\beta=5$ and $\beta=2$ for the two groups. In the "spacing-fair" case, the values of $T$ were set to $T=7$ and $T=20$, referring to the slot time of the user links at $150 \mathrm{Mbit} / \mathrm{s}$, while in the "delay-fair" case, the values of $T$ were determined so as to obtain an aggregate ASCDJ equal to about 180 and 750 for all VCs.

The coefficient of variation of the ASCDJ on the different VCs show, as expected, that the "bandwidth-fair" solution provides very significant differences in cell delay jitters, which can become extremely high for the VCs with low bit rate. The "spacing-fair" solution yields much smaller coefficient of variation of the ASCDJ on different VCs, but the unfairness of the shaping function is now reversed: the higher the bit rate of the VC, the longer the time spent in the shaper queue. Finally, the "delay-fair" case yields almost identical delays for all shapers. This result derived from the balance between the smaller spacing parameter $T$ and the higher probability of finding other cells in the shaper queue upon arrival for the VCs with higher average bit rate.

\section{Conclusions}

A simulation approach was used to investigate and compare the impact of two different traffic shaping policies on the QOS of CL services in two ATM network topologies. The considered shaping policies were an adaptation of the Generic Cell Rate Algorithm, and a Token Bucket Algorithm. The two network topologies are possible candidates for an Italian and a Hungarian ATM network.

The architecture of the considered traffic shaping device assumes that the shaping algorithms are applied to the traffic referring to groups of VCs. When more than one VC belongs to a group, a multiplexing of the VCs into groups is necessary before shaping. A further multiplexing of the shaped cell streams is necessary before crossing the UNI when more than one group is defined at a user.

The performance comparison was based on cell loss probabilities, cell delay jitter distributions, and number of cells brought to destination that must be discarded because some other cells of the same message were lost.

Numerical results showed that the way in which VCs are grouped is extremely important: the larger the number of groups, the better the performance improvement in terms of cell loss probability, but the larger the price to be paid in terms of user-to-user delay jitter.

Such result is quite intuitive, since controlling individual VCs avoids resource sharing among VCs in the same group, which can result in the use of a very high bandwidth by individual VCs for small periods of time. This may be a good result from the point of view of the cell delay, but is surely contrary to the traffic shaping philosophy.

It should also be observed that grouping has an effect on the ordering of cells: while cells within a group cannot overtake each other, the FIFO policy is not preserved among different groups.

The results that were presented assume a specific architecture for the traffic shaping device. Several different architectures may be devised for the implementation of the traffic 
shaping functions, and their performances may significantly differ from those presented in this paper.

As a final consideration we wish to observe that while a large number of groups is beneficial in terms of cell loss probability, a small number of groups is beneficial in terms of cell delay jitter. The adoption of either one of the two alternatives for those services that are most sensitive to either performance parameter seems interesting, but the effectiveness of the combination of the two approaches inside one network requires further investigations. Similarly interesting appears the investigation of the conditions under which the cell loss probability within the network is guaranteed to be zero, as a result of the low link loads and the quasi-deterministic traffic characteristics.

\section{REFERENCES}

1. M.Ajmone Marsan, R.Lo Cigno, M.Munafò, A.Tonietti, "Simulation of ATM Computer Networks with CLASS", Proceedings of the 7th International Conference on Modelling Techniques and Tools for Computer Performance Evaluation, Vienna, Austria, May 1994

2. ITU-T Recommendation I.371 "Traffic Control and Congestion Control in B-ISDN", Geneve, Switzerland, 1992

3. J. Turner, "New Directions in Communications (or Which Way to the Information Age?)", IEEE Communication Magazine, October 1986, Vol.24, n.10

4. M.Sidi, W.Liu, I.Cidon, I.Gopal, "Congestion Control Through Input Rate Regulation", IEEE Globecom 1989, Dallas, USA, Nov. 1989

5. A.E. Eckberg, D.T. Luan, D.M. Lucantoni, "Meeting the Challenge: Congestion and Flow Control Strategies for Broadband Information Transport", IEEE Globecom 1989, Dallas, USA, Nov. 1989

6. E.Wallmeier, T.Worster "On the Spacing Policer, an Algorithm for Efficient Peak Bit Rate Control", submitted for publication

7. P.Boyer, F. Guillemin, M.J.Servel, J.P Coudreuse: "Spacing cells Protects and Enhances Utilization of ATM Network Links", Network Magazine, Sept. 1992

8. F.Guillemin, P.Boyer, L.Romoneuf: "The Spacer-Contoller: Architecture and First Assessments", Broadband Communications 1992 IFIP, Elsevier Nort-Holland, 1992

9. F.Guillemin, W.Monin: "Management of Cell Delay Variation in ATM Networks", INFOCOM'g2,, 1992

10. J. Roberts, "Resource Sharing in B-ISDN Using Virtual Spacing Scheduling," Networks' 94, Budapest, 1994.

11. T. V. Do, L. Jereb, Gy. Wolfner: "Performance analysis of connectionless users with shaping", to be submitted to Management Committee Meeting of COST 242, Cambridge, UK, January 1995. 